\title{
Kernos
}

Revue internationale et pluridisciplinaire de religion grecque antique

19 | 2006

Varia

\section{Mythologia, Genealogia, Archaiologia}

Fonction paléontologique de la mythographie

\section{Françoise Graziani}

\section{(2) OpenEdition \\ 1 Journals}

Édition électronique

URL : https://journals.openedition.org/kernos/450

DOI : $10.4000 /$ kernos.450

ISSN : 2034-7871

Éditeur

Centre international d'étude de la religion grecque antique

Édition imprimée

Date de publication : 1 janvier 2006

Pagination : 201-214

ISSN : 0776-3824

\section{Référence électronique}

Françoise Graziani, « Mythologia, Genealogia, Archaiologia », Kernos [En ligne], 19 | 2006, mis en ligne le 21 mars 2011, consulté le 24 août 2022. URL : http://journals.openedition.org/kernos/450 ; DOI : https://doi.org/10.4000/kernos.450 


\title{
Mythologia, Genealogia, Archaiologia : fonction paléontologique de la mythographie
}

\begin{abstract}
Résumé : Les premières mythographies de l'Europe néo-latine, depuis la Genealogia deorum de Boccace, considèrent l'histoire des dieux sur le modèle des généalogies humaines, en cherchant à recomposer "la lignée de Saturne ». Les premiers historiens de la Grèce, comme les poètes, inventèrent des généalogies mythiques pour inscrire l'origine des hommes dans l'histoire de leur relation aux dieux. Que fondent les généalogies divines ? Non seulement des structures religieuses, non seulement la raison même des sociétés humaines, mais encore la préhistoire de l'humanité. Mais si les engendrements divins donnent sens aux relations entre les hommes et les dieux, c'est l'histoire des commencements du monde que les mythographes cherchent à comprendre en interrogeant l'articulation des généalogies divines et humaines. Depuis que, dans le Timée, Platon a associé mythologia et genealogia comme deux modes de discours « archéologiques », les mythographes ont ouvertement revendiqué un savoir sur le monde qui se définit comme une synthèse des «sciences de la nature ».
\end{abstract}

Abstract: Mythologia, Genealogia, Archaiologia: A Paleontological Scope for Mythography. The first European mythographical treatises in the Middle Ages and Renaissance, since Boccaccio's Genealogia deorum, were determined by a genealogical method, looking at the history of the gods with human patterns and seeking the reconstruction of "Saturn's lineage". The early Greek mythographers, like the poets, imagined various genealogies for seeking out the origins of humanity through their relationships with gods. What is founded on divine genealogies? Not only religious structures, nor only the true ratio of human sociability, but a human paleontology too. By interpreting filiations as a system of connexions between gods and humans, the mythographers aimed at understanding the protohistory of the universe. Since Plato's Timaeus associated mythologia and genealogia as two modes of "archaeological" discourse, mythographers have wanted to assert their own "physical science" as a whole synthetic perception of the natural world.

Le point de vue des mythographes de la Renaissance sur les savoirs antiques est aujourd'hui méconnu. Il s'agit pourtant de bien autre chose que de «l'ensemble des idées reçues sur les divinités du paganisme » divulguées depuis le XIX ${ }^{e}$ siècle par ces manuels scolaires dont Marcel Detienne a déploré le statut persistant de «modèle culturel » désuet ${ }^{1}$. Car les humanistes étaient familiers non seulement des textes poétiques et philosophiques les plus anciens, non seulement des pratiques herméneutiques les plus complexes des commentaires hellénistiques, mais aussi d'un lexique et d'un mode de pensée dont la fonction est désormais oubliée et dont ils tinrent en quelque sorte le registre.

\footnotetext{
${ }^{1}$ M. Detienne, L'invention de la mythologie, Paris, Gallimard, 1981, p. 238.
} 
Depuis Boccace, qui entre 1360 et 1375 composa la théogonie des temps modernes, les humanistes pratiquaient la mythographie dans une perspective à la fois rétrospective et syncrétique. Ils lui donnaient pour fonction d'opérer une synthèse entre poésie, sciences et religions, en incluant les pratiques religieuses et en poussant leurs investigations jusqu'à la perception de l'espace et celle du temps, jusqu'à s'interroger sur les âges du monde, sur la mesure de l'année, la succession des heures et l'équilibre des saisons. Ils avaient observé que pour les anciens déjà la mythographie était une remontée du temps: de la succession des générations humaines à la «lignée de Saturne $^{2}$ », de l'âge de fer à l'âge d'or, de la «théogonie» à la «cosmogonie ».

Dans l'Anthologie de Planude et dans la notice consacrée par Photius à Apollodore, ils pouvaient lire cette épigramme qui, dans le style équivoque des alexandrins, définit «non sans finesse » les objectifs de l'ancienne mythographie $^{3}$ :

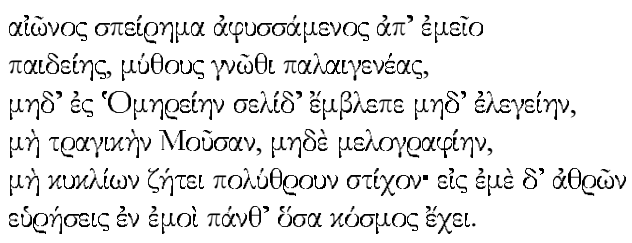

En remontant la spirale du temps éternel, apprends

de ma science les mythes qui viennent du lointain passé,

ne cherche pas dans les pages homériques ni dans les élégies,

ni du côté de la Muse tragique ou lyrique,

ne fouille pas les vers pleins de bruit des cycliques:

si tu regardes bien, tu trouveras en moi tout ce que le monde contient.

Le caractère publicitaire de cette annonce peut en masquer la portée théorique : en mettant en concurrence la «science» du mythographe (certes inscrite dans la pratique de la paideia, mais néanmoins revendiquée comme connaissance) avec celle des genres poétiques, comme si elle en faisait partie, elle fonde sa spécificité sur un projet «encyclopédique»: elle revendique une maitrise totale de modes de connaissance qui sont ceux des poètes, mais que ceux-ci énoncent séparément parce qu'ils doivent choisir des niveaux de discours différents, entre lesquels le mythographe opère une synthèse. Mais la fonction propre de la mythographie, en tant que point de vue sur le monde, semble bien être la « remontée du temps ». Dans sa brève présentation de la

\footnotetext{
${ }^{2}$ Tel est le titre du traité en langue française de Jean THENAUD, rédigé avant 1515 pour la formation intellectuelle du futur François 1er, et qui traduit partiellement Boccace (La lignée de Saturne ou Traité de science poétique, texte édité par G.M. MASTERS, Genève, Droz, 1973). J'utiliserai ci-après cette adaptation, en alternance avec des citations de l'original latin, comme témoin de la réception de Boccace au début de la Renaissance.

${ }^{3}$ Photius, Bibl., 142a-b. Je remercie Giovanni Tosetti d'avoir attiré mon attention sur cette épigramme, à l'occasion de sa communication de Bruxelles (voir ici même, p. 113-130). Toutes les traductions non identifiées sont miennes.
} 
Bibliothèque d'Apollodore, juste avant de citer l'épigramme, Photius insiste sur cette orientation " archéologique » ${ }^{4}$ :

Ce petit livre contenait les choses les plus anciennes (ta palaitata) de la Grèce, celles que le temps (ho chronos) leur a enseigné à penser (doxazein edôken) sur les dieux et les héros, ainsi que le nom des fleuves, des pays, des peuples et des cités, et tout ce qui remonte (anatrechei) aux origines (eis to archaion) [...] et redescend (kateisi) jusqu'à la geste troyenne [...] et les errances d'Ulysse sur qui s'achève l'exposé des temps archaïques (hê archaiologia). Ce livre est pour l'essentiel une vue d'ensemble (synopsis), et n'est pas sans utilité pour ceux à qui il importe d'avoir en mémoire les choses du passé (ta palaia).

Non seulement la mythographie enseigne à remonter le plus loin possible dans le passé des hommes et des dieux, mais elle parcourt « la spirale du temps » dans les deux sens, en avant et en arrière, pour rassembler le plus grand nombre possible de données doxographiques, historiques et cosmographiques. Pour les rhéteurs anciens, son efficacité ne se limitait donc pas à " apprendre à lire les poètes ${ }^{5}$ », elle formait l'esprit à penser le rapport entre l'espace et le temps à partir du mouvement «cyclique » qui accorde l'univers, les hommes et les dieux, pour retrouver une «antiquité » à laquelle le présent n'accède plus.

Aux XVI et XVII ${ }^{e}$ siècles, les premiers éditeurs des anciens mythographes grecs et latins ${ }^{6}$ leur reconnaissaient un statut de conservateurs du passé de l'humanité, non seulement parce qu'on y retrouve, sous forme de fragments, nombre de textes poétiques et philosophiques perdus, mais aussi parce qu'ils reconstruisent un savoir ancien sur les « origines » qui mettait en corrélation théogonie et cosmogonie et dont la cohérence est perdue ou en voie de l'être. Si Boccace peut être considéré comme un des fondateurs de la Renaissance humaniste, c'est d'abord pour son œuvre de mythographe, que lui-même définit métaphoriquement comme une recherche des «fragments dispersés par les anciens naufrages ». Collecter (colligere) les «reliques» du passé équivaut pour lui à une «exploration de la pensée des anciens", qui se trouve désarticulée et déposée, à l'état de ruines, dans les livres passés et présents, afin de chercher à comprendre « les significations cachées » sous les représentations figurées de dieux dont la «frivolité », la «consanguinité » et les «affinités» ne sont que des métaphores destinées à «couvrir d'un merveilleux mystère les choses de la nature ${ }^{7} »$. Le modèle généalogique est déjà en soi une herméneutique : en tête de chacun des treize livres qui forment le «corps » de sa mythographie, Boccace demande que soit dessiné

${ }^{4}$ Ibid.

5 Plutarque, Mor., 14d-37b.

6 Apollodore, Hygin, Palaephate, Fulgence et tous les mythographes anciens réunis en recueils, ont été édités plusieurs fois aux XVI et XVII siècles, dans toute l'Europe. Un des plus complets est le recueil de GALE, en deux volumes: Opuscula mythologica, physica et ethica, graece et latine ... in lucem edita per T. Gale, Cambridge, 1671 et Historiae Poeticae scriptores Antiqui, Graece et Latine, Paris, 1675.

BOCCACE, Genealogia deorum I, Proemium I, 40-46 (éd. ZACCARIA, Milano, Mondadori, 1998). 
un arbre où sont représentées les «générations » successives de dieux dont l'homonymie révèle la polysémie, et il laisse clairement entendre dans ses commentaires que l'image de l'arbre, dont une des variantes est la métaphore du corps démembré d'Hippolyte reconstitué par Esculape ${ }^{8}$, n'est pas pour lui une simple convention, mais qu'on doit l'entendre symboliquement, dans la perspective centrale selon laquelle il interprète les filiations et les noms divins, en termes d'allégorie naturelle. L'arbre n'est pas seulement une forme classificatoire, et en tant que telle mise en ordre des «affinités » qui donnent sens à la multiplicité des dieux du polythéisme païen : c'est proprement une image, allégorie de la matière première (bylê) qui, une fois organisée en forme (eidos), définit selon la physique aristotélicienne «l'origine » (archê) commune à toutes les productions de la nature. La collection de mythes, recomposés en un système généalogique dont l'arbitraire est ouvertement revendiqué , permet de «réduire en un corps» non seulement la multiplicité des informations contradictoires morcelées par le «naufrage » des œuvres du passé, mais aussi la «polysémie » ${ }^{10}$ inhérente aux figures et aux fonctions divines. La généalogie des dieux, imaginée par Boccace à la manière des poètes, se donne explicitement pour une recherche de sens : « en remontant la spirale du temps », elle porte sur le monde un regard «synoptique » pour saisir à la fois le moment de sa formation et celui de l'invention des dieux, inséparable de la prise de conscience par les premiers hommes de leur relation à la terre et au ciel.

Les premières mythographies humanistes, celles qui se conforment plus ou moins au prototype de la Genealogia deorum de Boccace, sont encore des théogonies. Tout en actualisant les anciennes « collections » hellénistiques et romaines de «vieux mythes », elles se souviennent des premiers « historiens » de la Grèce, tel Hécatée "qui organise les mythes du passé » (mythôn archaiôn synthetês ${ }^{11}$ ) en composant des généalogies mythiques pour chercher l'origine des hommes dans l'histoire de leur relation aux dieux. Il nous est difficile aujourd'hui de comprendre ce que les anciens entendaient dans ce contexte par syngraphê, synthesis et syntagma, qui semblent être employés partout comme des synonymes quand il s'agit de spécifier le mythologein du mythographe comme du poète. Même le périégète Pausanias, qui articule

8 «Je m'emploie à recueillir çà et là un corps de dieux et de héros païens, un corps qui fut grand mais qui maintenant est démembré, déchiré et presque réduit en cendres : et moi, nouvel Esculape, je m'apprête à le reconstituer, comme il a fait du corps d'Hippolyte » (ibid., 50).

9 «Les épaves que j'aurais réussi à trouver, je les ferai entrer [...], en les mettant en ordre comme je pourrai, dans la forme unifiée d'une généalogie, même si elles ont été disloquées, brisées et presque détruites par le temps. » (ibid. 40). Et plus loin, à propos de l'interprétation : « je transcrirai autant que possible les notations que j'aurai tiré des anciens, mais quand elles feront défaut ou quand, à mon avis, elles auront moins de pertinence, j'y adjoindrai ma propre opinion : et je le ferai avec la plus grande disponibilité d'esprit. » (ibid., 44).

10 «Il faut savoir que sous les fictions il n'y a pas une seule interprétation possible, mais on peut plutôt la dire polisemum, c'est-à-dire ayant plusieurs significations. » (o.c. I, iii, 7)

${ }^{11}$ Élien, Hist. animal. IX, 23 (cité par M. DETIENNe, o. c. [n. 1] p. 139, avec une autre traduction). 
dans le corps d'un livre la géographie physique de la Grèce, l'origine des toponymes, les mythes et les monuments fabriqués par les hommes, prend soin de définir son ouvre exégétique et descriptive comme syngraphê, en l'opposant à ce qui ne serait qu'un katalogon remémorant des noms ${ }^{12}$ : cette distinction, assez énigmatique pour nous, semble communément admise dans la langue grecque, et les humanistes la connaissent encore ${ }^{13}$. La différence entre les deux opérations semble être que le catalogue détache d'un ensemble déjà organisé des séries de noms ou d'objets divers, en les isolant pour les juxtaposer en une forme discontinue qui ne compose pas "un seul corps », alors que la syngraphê, comme la synthesis, combine des données éparses en les articulant pour les rendre signifiantes. Chez Boccace, les verbes colligere et componere sont sans cesse employés l'un et l'autre pour décrire le travail du mythographe : construire des systèmes de relations entre les noms des dieux et les «principes naturels » qui y sont cachés, entre l'interrogation sur le passé et l'enseignement qu'il est possible d'en tirer au présent et au futur. Dans le latin de la Renaissance, hérité de la scolastique médiévale, le mot compositio est aussi précis que son pendant expositio, qui nomme la description et l'interprétation des mythes et des «contenus » cachés sous les mots. Dans le discours de l'exégète comme dans celui du poète la "composition », plus qu'une mise en ordre, est l'indice même de la cohérence de la pensée, et traduit exactement la synthesis (ou systasis) par quoi Aristote définit la fonction du mythos, et qu'il met en corrélation avec son statut d'archê quand il dit que «le mythe est le principe, et pour ainsi dire l'âme de la tragédie ».

En 1548, Lilio Gregorio Gyraldi, inaugurant une position critique envers la vieille théogonie de Boccace dont il juge les sources et la démarche trop peu fiables, se défend de proposer dans son Historia deorum une nouvelle

\footnotetext{
${ }^{12}$ Je remercie Vinciane Pirenne-Delforge d'avoir attiré mon attention sur cette déclaration de Pausanias, et de m'en avoir communiqué la référence (VI, 1, 2 et passim).

${ }^{13}$ Tiziano DORANDI (Le stylet et la tablette. Dans le secret des auteurs antiques, Paris, Les Belles Lettres, 2000, p. 84-86) rend compte d'une opposition de même type entre syntagmatikon et bypomnêmatikon, telle qu'elle est énoncée par les commentateurs alexandrins pour expliquer la méthode de travail d'Aristote : «quand on composait des écrits (sungrapsasthai) autrefois, on notait d'abord sommairement ses propres découvertes (ta beuriskômena) en vue de la démonstration de son propos, puis on recueillait beaucoup de pensées dans les livres du passé (archaioterôn bibliôn) afin de confirmer ce qui était correct et de réfuter ce qui ne l'était pas, et enfin on mettait le tout en ordre (taxin) et on ornait les compositions (ta sungrammata) de beau style et de mots choisis. Les mémoires (bypomnématika) diffèrent ainsi des compositions (syntagmata) par l'ordre et la beauté de l'expression (bermêneias)» (Ammonios, In Cat. Arist. p. 4, 3-13 Busse, c'est moi qui traduis). Dans la pratique en usage depuis l'époque hellénistique, il y avait donc, selon T. Dorandi, deux catégories d'écrits : les simples notes de cours ou de lecture, aide-mémoire non travaillés pour la publication, et les syngrammata qui sont des «compositions achevées » jugées publiables par leur auteur. Les humanistes connaissent bien cette distinction, qu'ils discutent souvent à propos du corpus aristotélicien, car les commentaires d'Ammonios, de Philopon, de Simplicius, ont été très largement diffusés et utilisés tout au long du Moyen-Age et de la Renaissance.

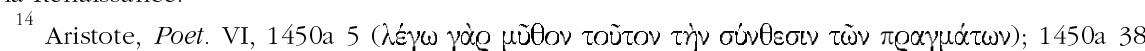

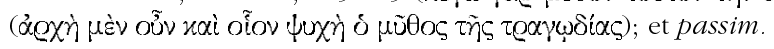


genealogia : et comme pour confirmer cette revendication, il donne à chacun de ses chapitres le titre de Syntagma, résumant d'un mot le programme annoncé dans la dédicace, qui signale que son «histoire des dieux » entend construire des connexions polyvalentes entre les noms, les images, les attributs divins et la localisation géographique des cultes, rites et cérémonies religieuses ${ }^{15}$. Cette opposition nouvelle entre genealogia et syntagma suggère que, pour Gyraldi comme pour tous ceux qui par la suite vont adopter son point de vue en niant la cohérence du classement généalogique ${ }^{16}$, les généalogies des premiers mythographes sont indissociables d'une conception «archaïque » des rapports entre mythe, littérature, religion, discours exégétique et étiologie. Ce clivage ne fera que s'aggraver au cours de l'histoire de la «science mythographique», jusqu'au point de rupture marqué par le XVIII siècle.

En 1783, en présentant son édition de la Bibliothèque d'Apollodore, dont il loue «la clarté de la mise en ordre " (ordine lucido), Christian Gottlob Heyne s'emploie à définir une frontière bien nette entre les «mythes des poètes » et ceux de la tradition exégétique, qu'il accuse d'être source de confusion et d'avoir empêché les «mythes littéraires» de parvenir «intacts» (intactos) jusqu'aux temps modernes, ayant été «changés et renouvelés » (mutarunt ac novarunt) par tous les «Chronographes, Géographes, Astronomes, Archéologues (Archaeologi), Naturalistes (Physici) et Philosophes» qui les ont encombrés d'interprétations multiples et équivoques ${ }^{17}$. Le mérite d'Apollodore serait pour lui d'avoir clarifié la matière obscure qu'il a rassemblée, notamment en réservant l'examen des «théogonies et cosmogonies» aux seuls premiers chapitres de son livre, alors que les "premiers historiens, qui de notre jugement devraient plutôt être appelés mythographes », les rhéteurs alexandrins et leurs épigones (dont Diodore de Sicile) leurs consacraient des volumes entiers. En retraçant l'histoire de la tradition mythographique, Heyne ne reconnaît plus la légitimité des généalogies mythiques que dans la perspective d'une recherche des « origines » nationales, de la fondation de peuples ou de cités.

Mais les premiers mythographes humanistes, peut-être parce qu'ils avaient conscience de commencer un nouvel âge historique où se posait la nécessité de refonder une «science de l'antiquité », ont refait intégralement le chemin des origines en recombinant la théogonie, la cosmogonie, la cosmographie

15 Lilio Gregorio GYRALDI, De deis gentium libri sive Syntagmata XVII, quibus varia ac multiplex Deorum Gentium Historia, Imagines ac Cognomina, plurimaque simul multis hactenus ignota explicantur, clarissimeque tractantur, Bâle, $1565^{2}$ [1548], p. 5 (non genealogias Deorum dico, sed et nomina et cognomina, effigiesque, insigniaque, et quae patria cuique est, sacra quoque atque caeremonias).

6 À partir surtout de Cartari et Conti, dont l'agencement est thématique. Le classement alphabétique des dictionnaires de mythologie est la forme de taxinomie la plus moderne.

17 Apollodorus Atheniensis Bibliothecae libri tres et fragmenta, curis secundis illustravit C.G. Heyne, Hildesheim / New York, Georg Olms Verlag, 1972, facsimil. de l'éd. de Göttingen, 1803, p. xl-xli (préface datée de 1783). 
(géographie et cosmologie mêlées), la généalogie des héros et des hommes, la mesure du temps humain (chronologia), la philosophie naturelle (physiologia), bref tout le savoir du monde, condensé dans la perspective synthétique d'une archaiologia. Ils n'avaient pas encore oublié que les antiques généalogies et les «chroniques » d'une histoire qui se voulait « universelle » travaillaient à construire des filiations entre les dieux et les hommes pour instaurer entre eux une dépendance «naturelle ». Pour les humanistes comme pour les « conteurs de mythes» (mythologoi) antiques, la «science » mythographique réactive une prisca sapientia qui se confond avec ce que Platon définissait précisément comme «recherche du temps perdu» (anazêtêsis tôn palaiôn) et que, à la suite des anciens «sages», il appelait archaiologia ${ }^{18}$.

La généalogie de Boccace, nourri de sources patristiques et médiévales mais qui cite volontiers Aristote, Platon, Plutarque et un grand nombre d'auteurs plus rares, est une encyclopédie qui recompose l'histoire du monde à travers la reconstitution de «la lignée de Saturne ». Mais Boccace remonte plus haut que Saturne, car le plus «vieux ${ }^{19}$ » des dieux, le premier à avoir un nom et une histoire, est aussi précédé du Ciel et de la Terre, et c'est pourquoi, dans sa recherche conjointe des commencements du monde et des origines de la pensée religieuse, Boccace « invente » un dieu inengendré, coextensif à l'Éternité et contemporain du Chaos, qu'il appelle Demogorgon : ce dieu inconnu, si «archaïque » qu'il est sorti de la mémoire des hommes, au nom si «terrifiant» que les anciens poètes ont préféré le taire, Boccace déclare le connaître par les mythographes et semble ignorer son homonymie avec le dêmiourgon du Timée ${ }^{20}$. Le dieu-artisan qui crée le monde, et dont

\footnotetext{
${ }^{18}$ Le Timée, qui expose les ambiguités des liens entre mythologein, genealogein et archaiotata legein (22a-23b), et le Critias (mythologia gar anazêtesis te tôn palaiôn, 110a) identifient clairement mythologia et archaiologia. Cf. M. DETIEnNE, o.c. [n. 1], p. 163-167; et L. BRISSON, Platon, les mots et les mythes, Paris, Maspéro, 1982, p. 44-46 et 189-190.

19 "Saturne est mis le premier des dieux, triste, vieux, chenu, ayant la tête couverte, dévorateur de ses enfants. [...] Non improprement il est peint vieux, car vieillesse est la voisine de mort. [...] Souvent les philosophes prennent Saturne pour le temps et mêmement les Grecs qui le nomment Cronon, c'est-à-dire le Temps, et disent qu'il a la tête couverte parce qu'on ignore quel a été le commencement du temps. " (Thenaud, o.c. [n. 2] I, i-iv, p. 61-62; orthographe modernisée dans toutes les citations). Cf. BoccACE, Genealogia deorum VIII, 1.

${ }^{20}$ Boccace cite pour source un certain Théodontius, qui ne nous est connu que par lui, et qui aurait commenté une glose de Lactance Placide à la Thébaïde de Stace (dicit autem deum demiourgon, cujus scire non licet) à propos d'un dieu au nom imprononçable invoqué par Tirésias dans une séance de nécromancie, où il «ouvre le chaos infernal» pour faire sortir les ombres du centre de la terre (IV, 515-516). Le dieu terrifiant de Boccace correspond aux paroles de Tirésias : « je pourrais faire trembler Hécate... et le souverain du triple monde qu'il est interdit de connaître, mais je me tais ». Les commentateurs, depuis Gyraldi, se sont mépris sur les raisons de l'intérêt de Boccace pour ce nom qui n'apparaît dans aucune source ancienne, et qu'on attribue à un «barbarisme». Bien que Boccace sache peu de grec, il a reçu l'enseignement direct, à Florence, de Léonce Pilate et connait très certainement le Timée, qu'il cite ailleurs et qui fut le dialogue platonicien le plus commenté au Moyen Age. Même si le rapprochement entre le dieu « inconnaissable » de Stace et le «démiurge » de Platon vient de Lactance, et même si le mot a été déformé par un autre, le choix de ce nom n'est pas fortuit : on a prêté à Boccace une
} 
Platon parle «à l'imparfait ${ }^{21} »$, semble le plus qualifié, parce qu'il est antérieur au temps historique et qu'il ressemble au Dieu biblique, pour être l'ancêtre commun à tous les dieux. Mais si Boccace ignore ici Platon, c'est que son Demogorgon ne se confond précisément pas avec le Dieu créateur de l'harmonie cosmique, il est au contraire le dieu des orages, des catastrophes sismiques et de la nature discordante. L'invention de ce dieu "archaïque » répond à une nécessité heuristique, et c'est pourquoi il intervient dans le prologue, antérieurement à tout « ordre » généalogique. Boccace déclare avoir «cherché le dieu le plus antique» dans les opinions des anciens physiologoi qui, comme les anciens "poètes théologiens », identifiaient les dieux primitifs avec les «causes premières du monde », mais Thalès, Anaximène, Chrysippe, Alcméon de Crotone, et aussi Macrobe, ont attribué l'origine de toutes choses à l'eau, à l'air, au feu, au soleil ou aux corps célestes. Boccace préfère l'hypothèse de Théodontius, mythographe inconnu présenté comme «un moderne qui a cherché avec une attention particulière à comprendre ce genre de choses», parce qu'il rapporte l'opinion «des plus anciens hommes d'Arcadie » qui attribuaient à la terre l'origine de toutes choses ${ }^{22}$. Demogorgon, le seul dieu qui puisse être déclaré «non père du monde, mais de tous les dieux païens », est logé sur terre dans l'antre de l'Eternité, car c'est du centre de la terre que le passé rend ses oracles, et Boccace interprète son nom comme signifiant étymologiquement « dieu de la terre, ou plutôt sagesse (sapientia) de la terre, car demon signifie sapience ou science $»^{23}$. Dans le nom du dieu qui doit symboliquement engendrer la lignée de Saturne, Boccace reconnait celui de la Gorgone, dont il fait une des Furies infernales et qu'il associe étymologiquement à Gaia, la terre, mais aussi un « démon», et il a pu lire dans Plutarque que la race (genos) des démons est préposée aux oracles $^{24}$ et chez Rémi d'Auxerre que le mot daêmôn signifie «savant en toutes choses $»^{25}$. La «sagesse de la terre » n'est autre que cette connaissance interdite aux hommes et antérieure aux dieux mêmes, que le mantis et le

crédulité et une ignorance dont il est très éloigné, sans accorder crédit au caractère délibéré de son « invention », qu'il a pourtant longuement justifiée. C'est précisément parce qu'il n'existe pas que Boccace s'intéresse à ce dieu, et qu'il compose un mythe sur son nom.

${ }^{21}$ Plotin, Ennéades III, 5, 9 (cité par Detienne, o.c. [n. 1] p. 235).

${ }^{22}$ Genealogia deorum I, Proem. II, 1-15.

23 id. Proem. III, 11.

${ }^{24}$ De defectu oraculorum, 13-21 (Mor., 416b-421b). Plutarque définit les démons, « que Platon [Conv. 202e] appelle herméneutes », comme "périssables et imparfaits », ce qui les distingue des dieux dont ils adoptent souvent les noms. Ils ont pour domaine le sensible, c'est-à-dire « la nature qui gouverne destruction et génération, action et passion » (Mor. 394a), et ont donc souvent des influences néfastes sur les hommes : «les sacrifices rituels, les cérémonies religieuses et les mythes (mythologiai) en conservent des vestiges et des indices (ichnê kai symbola) dispersés un peu partout. [...] Tout ce que racontent les mythes sur les rapts commis par les dieux, leurs errances, exils et servitudes, sont des passions (pathêmata) et des aventures (tychê) qui concernent non les dieux mais les démons » (417b-e).

${ }^{25}$ Thenaud (o.c. [n. 2] II, i, p. 66) cite REMI D'AuXERRE (Commentaire à Martianus Capella II, 65.19) pour expliquer que les Démons sont fils de Rhéa, la Terre. L'association daimôn - daêmôn vient du Cratyle (398b). 
vates obtiennent en interrogeant le monde des morts. C'est pourquoi la Pythie appelle la Terre protomantis, et l'invoque, dans le prologue des Euménides, pour avoir été la première prophétesse de Delphes avant l'arrivée du dieu Apollon $^{26}$.

L'étroite relation ainsi instaurée entre «l'éternité » (l'aiôn des grecs), perceptible uniquement dans des pratiques oraculaires qui font coïncider le passé, le présent et le futur, et l'élément terre, le seul des principes élémentaires constituant la «matière» du monde physique qui soit habitable pour l'homme, est à mettre en relation avec la recherche «archéologique » propre au mythographe, et il faut d'abord l'interpréter " physiquement », mais sans perdre de vue la nécessaire «polysémie» de la méthode allégorique de Boccace. Car la mythographie de Boccace est une physiologia entendue à la fois comme science de la nature et comme paléontologie humaine; dans l'histoire des dieux elle cherche d'abord à comprendre les opinions et comportements «archaïques » des hommes, mais son exploration «archéologique » débouche aussi sur une anthropologie religieuse. C'est sans doute dans le même esprit que la Theologia mythologica de Georg Pictor, qui en 1532 commence son histoire des dieux avec Jupiter et les Olympiens, remonte jusqu'à la série des divinités chtoniennes : Janus, Saturne, Genius (dieu de la génération), Pluton, Bacchus et la Terre, réunis significativement à la série des bienfaiteurs de la race humaine (Hercule, Esculape, Flore, Eole, Priape, Cupidon, Volupté) qui leur fait suite ${ }^{27}$. Et Vincenzo Cartari, dont le propos n'est plus généalogique, commence pourtant ses Images des dieux par Saturne, en le faisant précéder de la description de l'antre de l'Eternité de Boccace, et le présente, en citant Virgile, comme le dieu civilisateur qui tira des cavernes les sauvages populations « indigènes » pour devenir, dieu grec exilé en terre italique, le premier roi de l'antique Latium ${ }^{28}$.

Pour tous les humanistes, la terre mythique par excellence est l'Arcadie, réputée plus ancienne que la Lune : c'est là que les poètes latins avaient situé l'âge d'or et le royaume de Saturne, et c'est là que Boccace situe l'habitat troglodyte des premiers hommes ${ }^{29}$. En Arcadie, les hommes ont pour ancêtres les dieux les plus «archaïques», Janus qui ouvre les portes du temps et qu'Ovide identifiait au $\mathrm{Chaos}^{30}$, ou Pan, le dieu de l'Univers qui gouverne la

\footnotetext{
${ }^{26}$ Eschyle, Euménides, 2-4

${ }^{27}$ Georg PICTOR, Theologia mythologica, Michaël Hillenius, Anvers, 1532, livres II et III

${ }^{28}$ Vincenzo CARTARI, Immagini degli dei degli antichi $\left[1571^{2}\right]$, a cura di C. VOLPI, Roma, 1996 p. 33-41 (cite Virgile, Enéide VIII, 319-325). L'antre de l'Eternité est encore la demeure de Démogorgon : tout en déclarant douter de son authenticité, puisqu'il ne le trouve dans aucune source ancienne, Cartari ne rejette pas le dieu proto-archaïque de Boccace, parce qu'il s'accorde avec la recherche des origines du monde qui détermine toute mythographie.

29

«En celuy temps les hommes et femmes qui n'avaient cités ni maisons demeuraient ès fosses de la terre et ès arbres cavés (...) De ce procède que l'on trouve écrit les Arcades avoir été avant la Lune, planètes et étoiles, car leur demeurance fut par long temps ès spélonques et fosses de la terre opaque et ès forêts ténébreuses. » (Thenaud, o.c. [n. 2] I, 10-11, p. 65). Cf. Ovide, Fastes I, 469-470; II, 290; V, 90.

${ }^{30}$ Ovide, Fastes I, 103
} 
Nature primitive et dont le corps est un composé de terre et de ciel ${ }^{31}$. C'est là que, selon Boccace, les hommes du temps des cavernes attribuèrent «la sapience divine » à Demogorgon, tant ils étaient effrayés par les tremblements de terre et les éruptions volcaniques. Et parce que «les antiques arcadiens de ce lointain passé pensèrent que le silence devait accroître la majesté de son nom, ou estimèrent qu'il était inconvenant qu'un nom si sublime soit prononcé par la bouche des mortels, ou eurent peur que le dieu nommé s'irrite contre eux », pour cette raison son nom est resté inconnu, oublié pour n'avoir pas été transmis dans la mémoire des hommes ${ }^{32}$.

Selon la tradition poétique latine, c'est donc avec Saturne que commence le «catalogue » des dieux et le temps des hommes ${ }^{33}$. Le plus «vieux» des dieux connus, assimilé par les mythographes à Chronos, est aussi une figure de la sagesse divine, et c'est pourquoi Boccace admet qu'il lui revient d'engendrer les quatre éléments qui, une fois mis en ordre, formeront le $\operatorname{cosmos}^{34}$ :

Saturne, interprété sacré sens, est pris pour la divine sapience et providence, de laquelle toutes choses sont procréées. Car de lui sont descendus Jupiter, Juno, Neptune, Pluto ou Dis, par lesquels sont entendus les quatre éléments [feu, air, eau, terre]. Il est nommé fils de Polure, c'est à dire de celui qui a plusieurs enfants.

Il n'est ni contradictoire ni incongru que le traducteur de Boccace attribue à ce Saturne père des éléments un père inconnu dont seul le nom, interprétable comme «celui qui a beaucoup d'enfants», justifie l'antériorité : cette attribution a pour fonction de compléter une archaiologia à laquelle semble manquer la preuve étymologique, puisque le nom latin de Saturne ne dit rien de sa nature chtonienne archaïque. C'est dans la même perspective symbolique que certaines divinités non engendrées n'ont pas de descendance, à la manière de l'Éros primordial qui, selon Hésiode, tire le monde du chaos et que Platon déclare inengendré ${ }^{35}$. Telle est, chez Boccace, Athéna, autre figure

\footnotetext{
31 «Selon Varron [...] Pan, qui est interprété Tout, est peint avec cornes semblables aux rais de la Lune et du Soleil [...] En sa poitrine sont les signes [...] du Zodiaque. Dès le nombril en bas est tout velu comme un ours ou lion pour les arbres, herbes et bêtes. Il a pieds de chèvre qui représentent la stabilité de la terre. [...] Les sages ont dit que Pan était bylè, c'est-à-dire la matière confuse de laquelle premièrement les éléments, puis les choses élémentées ont été faites et formées. » (THENAUD, o.c. IX, 2, p. 109)

32 Genealogia deorum I, Proem. III, 5-7

33 «Il est dit l'origine des dieux pour ce que ses fils et filles ont été mis au catalogue des dieux poétiques » (ThENAud, o.c., version abrégée, l.c. p. 51)

${ }^{34}$ ThenAUD, o.c. III, 1, p. 69; traduit Genealogia deorum VIII, 1, 32 : Saturnum quasi sacrum nun; nus enim grece sensus dicitur, aut satorem nun, quasi divinum sensum creantem omnia. L'étymologie et la relation à «Polure » viennent de Fulgence, Mythologiae 1, 2.

${ }^{35}$ Platon, Banquet, 178 b, discours de Phèdre sur l'Éros d'Hésiode, de Parménide et d'Acousilaos («le signe qu'Éros est le dieu le plus ancien, c'est qu'il n’a pas de parents : aucun logographe ni aucun poète ne lui en attribue »).
} 
de la «sapience divine» qui, parce qu'elle détermine toute connaissance, échappe à l'ordre naturel des générations ${ }^{36}$ :

Cette Pallas est sans mère, car sapience n'a ni commencement ni fin. Elle est vierge, car elle ne reçoit aucun vice ou corruption, mais est ornée de perpétuelle intégrité de mours. (...) Le nombre septenaire lui est sacré, car c'est le nombre seul au dedans de dix qui n'engendre ni ne produit autre nombre, et n'est produit que d'unité qui est la mère et racine de tous nombres.

Ainsi, bien que la "généalogie des dieux » soit conçue sur le modèle des généalogies humaines, avec de père en fils la même récurrence de patronymes, on ne peut se contenter de l'interpréter, en termes d'allégorie historique ou evhémériste, comme la divinisation de quelques « héros » humains. Malgré ce modèle humain, l'histoire des dieux reste en effet fondamentalement liée à celle du cosmos, elle dépasse originairement l'ordre humain, même si elle finit par le rejoindre dans les engendrements hérö̈ques, pour articuler les quatre éléments qui précèdent, au commencement des temps, l'union du ciel et de la terre.

L'articulation dialectique entre le temps proto-historique des dieux (aiôn) et le temps historique des hommes (chronos) se trouve mise en scène dans le cycle des anciennes généalogies, lorsque la recherche des «antiquités divines » conduit à interpréter l'origine " physique » du monde, mais aussi à dater et à localiser la présence des hommes sur la terre. De ce fait, cette archaiologia ne peut manquer de s'intéresser aux métamorphoses et aux cataclysmes dont la terre et le ciel ont été le lieu, depuis que la discorde du chaos s'est changée en harmonie cosmique. La lignée de Saturne-Chronos, qui inaugure le temps des hommes et s'achève avec Hercule, n'apparaît dans la Généalogie des dieux qu'à partir du livre VIII, et n'occupe qu'un tiers des quelques huit cents pages qui constituent le volume. Les sept livres précédents recomposent la pré-histoire des dieux. Dans le prologue de ce huitième livre, Boccace s'attarde à développer la métaphore de la navigation, fiction poétique qui rythme de livre en livre sa traversée des temps mythiques : pour marquer le passage du temps des archai au temps humain, le mythographe se représente pris dans une tempête nocturne qui le plonge dans l'épouvante «à voir la nature si grandement transformée, comme si revenait l'antique Chaos ». Mais lorsqu'apparâit à l'horizon la planète Saturne, dont il connaît bien les propriétés, le navigateur solitaire reconnaît la stabilité relative de l'ordre du monde, et poursuit sa route.

Platon et Aristote l'ont dit et répété, ce n'est pas une fois mais plusieurs que le monde a été détruit par l'eau, le feu ou les tremblements de terre et qu'un nouveau monde s'est formé sur les ruines d'un passé oubliée ${ }^{37}$. Dans le Timée, Platon associait déjà mythologia et genealogia comme deux modes de discours «archéologiques » destinés à nous renseigner sur l'histoire des

\footnotetext{
${ }^{36}$ ThEnAUD, o.c. XI, ii, p. 127 (traduit Genealogia deorum II, 3, 6).

37 Platon, Timée, 22c-23b et Critias, 112a; Aristote, Met. XII, 8, 1074b; Du ciel I, 3, 279b; Meteor. I, 3, 339b.
} 
révolutions sismiques et la mort des civilisations humaines, en rapportant le récit d'un sage ancien, Solon. Le temps efface de la mémoire des hommes «les choses du passé le plus lointain (tôn archaiôn)», au point que les Grecs, de l'avis du sage égyptien qui donne à Solon une leçon d'archaiologia, « ne savent presque rien sur les choses du passé (ta palaia)». Solon, que les Athéniens considèrent comme le plus «sage » d'entre eux, est mis en présence d'un peuple «sage » qui partage avec lui le culte de la déesse Athéna parce que, sous un autre nom, elle est leur "ancêtre » commun. Les Grecs, bien qu'ils aient été « engendrés et éduqués par des dieux», n'ont d'autre antiquité à exposer (ta archaiotata legein) que des mythes: Solon parle (mythologei) de Phoronée, " qu'on dit être le premier homme », de Deucalion et Pyrrha qui, après le déluge, refondent une nouvelle humanité. Pour témoigner de son savoir archéologique et obtenir en retour des informations «sur les choses du passé », Solon «fait aussi la généalogie (genealogein) de leurs descendants » et calcule le temps (tous chronous) qui sépare le présent des origines mythiques de l'humanité. Mais il s'entend reprocher par le sage Égyptien de n'avoir «aucune vieille opinion (palaian doxan) transmise depuis l'antiquité (di' archaian) ni aucun savoir (mathêma) blanchi par le temps », en raison de tous les cataclysmes qui, depuis les origines, ont «plusieurs fois et de bien des manières détruit le genre humain ». Alors que les Athéniens, comme tous les autres peuples qui « repartent à chaque fois du début (ex archês)», sont donc «toujours jeunes (oion neoi gignesthe) » et « ignorants de ce qui est arrivé dans l'ancien temps (tois palaiois chronois) », la supériorité des sages Égytiens tient à un phénomène géologique qui leur a permis d'appréhender la continuité des temps et de « conserver par écrit dans les temples » toute l'histoire de l'humanité : le régime régulier des crues du Nil a toujours protégé l'Égypte des déluges aussi bien que des incendies catastrophiques. Les généalogies de Solon «diffèrent bien peu des mythes pour enfants (paidôn brachy ti diapherei mythôn)», parce que les grecs ont perdu la mémoire des temps antérieurs au seul déluge dont la trace subsiste encore dans leurs traditions orales, et parce qu'ils n'ont pas consigné par écrit l'histoire de la terre. Alors que le savoir «archéologique» des Égyptiens est tout à la fois, et irréductiblement, historique, scientifique et théologique : le sage qui révèle à Solon l'histoire de l'Atlantide (qui, si elle avait pu être écrite en grec, aurait concurrencé l'épopée homérique) lui apprend aussi que le peuple du Nil tient directement ses lois et sa science d'Athéna, comme ce fut autrefois le cas des Athéniens eux-mêmes. Il lui enseigne en outre que la déesse a choisi elle-même le site où fonder la ville d'Athènes, en fonction de «l'heureux mélange des saisons qui devait produire les hommes les plus intelligents (phronimôtatous)», pour que les citoyens d'Athènes puissent lui ressembler, en tant qu'elle est «la déesse qui aime la guerre et la sagesse (philopolemos te kai philosophos hê theos) ». La phronêsis qui aurait dû être celle des Athéniens, et qui est bien celle de leurs «parents » égyptiens, est très précisément décrite : ceux qui observent la loi de la déesse possèdent «à partir des commencements (kat'archas) un savoir total sur le monde (peri ton 
kosmon [...] mathêmata panta)" qui va de la mantique à la médecine et couvre toutes les sciences divines et humaines ${ }^{38}$.

La leçon donnée à Solon par l'Égyptien, qui le surpasse en sagesse parce qu'il connaît ta archaiotata et qu'il sait remonter jusqu'aux causes (aitia) et origines (archai) des vicissitudes de l'histoire humaine, est bien une leçon de sagesse «mythologique»: il lui enseigne à recouvrer la mémoire, en ne s'arrêtant pas au temps des récits mythiques mais en remontant jusqu'à l'histoire de la terre à travers les désastres successifs de l'humanité. Connaître la multiplicité et la répétition des séismes, déluges et incendies provoqués par les éléments, c'est être en mesure de connaître le monde en faisant la synthèse de toutes les sciences, cosmologie, astrologie, géologie, physiologie, médecine, anthropologie, histoire et géographie, et c'est par là aussi être théologien. C'est pourquoi la rencontre de Solon et du sage égyptien sert de prologue à ce qui est l'objet propre du Timée, une nouvelle cosmogonie.

De tous les thèmes ici condensés par Platon sous forme de conte philosophique et qui furent largement exploités et développés par la tradition mythographique, la relation entre histoire de l'humanité et histoire du monde demeure un des plus féconds, mais aussi un des plus secrets. Le mythe de Deucalion et Pyrrha et celui de Phaéton, dont Platon suggère qu'ils sont les prototypes d'une histoire des cataclysmes cosmiques auxquels tous les peuples humains ont été soumis, ne sont que des exemples partiels de la connaissance des «choses du passé » dont la généalogie et ses corollaires, l'allégorie naturelle, la chronologie universelle et l'histoire des inventions utiles à l'homme ${ }^{39}$, sont des approches propédeutiques. Il ne suffit pas de raconter des mythes, il faut savoir «remonter en arrière » pour reconnaitre (sous le «voile» qui cache leur signification par cette opération de l'esprit que Platon nomme byponoia et les mythographes allêgoria) une histoire qui relève autant de l'épopée des poètes «cycliques » que du catalogue géologique qui « raconte » les formations et destructions successives de la croûte terrestre. Cette recherche du passé (anazêtêsis tôn palaiôn), qui est bien autre chose qu'un dévoilement, est proprement la tâche que s'assignèrent les mythographes humanistes, dans le but de réaliser en quelque manière une synthèse entre la science universelle des Égyptiens et l'ignorance chargée de sous-entendus des mythes grecs, qui en disent plus qu'on ne croit.

Les questions que posent les mythographes n'ont pas encore été résolues : quel enseignement les généalogies des premiers « historiens » grecs, et les

\footnotetext{
38 Platon, Timée, 21e-24d (trad. L. BRISsON, Paris, Flammarion, 1992 - je modifie la traduction de la dernière citation : Luc Brisson choisit d'entendre peri ton kosmon en un sens taxinomique : «suivant cet ordre », tout en avouant ne pas bien comprendre la construction embrouillée de la phrase; je crois que la lecture en est facilitée si on l'interprète dans la cohérence d'un contexte à la fois cosmogonique et mythographique, qui attribue aux Égyptiens un «savoir sur le monde » revendiqué par toute la tradition sophistique).

39 Les logographes de la Renaissance complétèrent les traités mythographiques par des recueils spécialisés recomposant l'histoire de toutes les inventions. Le plus célèbre, le $D e$ inventoribus rerum de Polydore Virgile (Venise, 1499) traite aussi de l'origine des religions (I, 5 , 2) et contient une chronologie universelle.
} 
chronologies qui prétendent remonter sans solution de continuité jusqu'aux origines du monde, nous apportent-elles sur l'apprentissage de la connaissance et l'histoire de la pensée ? De quel savoir «fragmenté » les questionnements qui nous sont parvenus sous forme de mythes sont-ils le vestige ? Que signifie la répétition inlassable des mythes, toujours à réinterpréter depuis des temps immémoriaux ? Ne peut-on les lire comme le témoignage d'une antique «science de la nature », quand on sait que les anciens «sages» de la Grèce s'attribuaient à la fois les noms de physiologoi et de theologoi ? Seule aujourd'hui une perspective pluri-disciplinaire peut permettre d'affronter ces questions dans toute leur complexité, en articulant les données de la mythographie, de la philologie, de la paléontologie et de l'histoire des religions à ce «discours sur les dieux» que les anciens Grecs nommaient theologia.

Que fondent les vieilles théogonies? Non seulement des structures religieuses, non seulement la raison même des sociétés humaines, mais encore, par delà la préhistoire de l'humanité, l'histoire imaginée des mouvements et métamorphoses de la terre. Les engendrements divins donnent sens aux relations entre les hommes et les dieux, qui sont le fondement des religions, mais c'est l'histoire des commencements du monde et de ses transformations successives que les poètes et les mythographes cherchent à inscrire dans la continuité des temps en configurant sous forme de fictions le mouvement perpétuel des générations, analogue et contigu à la course des planètes dans le ciel et aux cycles naturels sur la terre.

Françoise GRAZIANI

Université Paris 8

Département de Littérature comparée

2, rue de la Liberté

F - 93526 SAINT-DENIS Cedex

Courriel : antgraz@club-internet.fr 